\title{
The Path to Actinorhodin: Regio- and Stereoselective Ketone Reduction by a Type II Polyketide Ketoreductase Revealed in Atomistic Detail
}

\author{
Stefano A. Serapian,,$^{\dagger} \wedge$ John Crosby ${ }^{\ddagger}$ Matthew P. Crump $₫$ and Marc W. van der Kamp, ${ }^{*, \dagger}$ \\ †School of Biochemistry, University of Bristol, University Walk, Bristol, BS8 1TD, United Kingdom \\ †School of Chemistry, University of Bristol, Cantock’s Close, Bristol, BS8 1TS, United Kingdom \\ KEYWORDS: Polyketide synthesis, Protein-protein Docking, Computational Enzymology, 2D-NMR, QM/MM
}

\begin{abstract}
In type II polyketide synthases (PKSs), which typically biosynthesize several antibiotic and antitumor compounds, the substrate is a growing polyketide chain, shuttled between individual PKS enzymes whilst covalently tethered to an acyl carrier protein (ACP): this requires the ACP interacting with a series of different enzymes in succession. During biosynthesis of the antibiotic actinorhodin, produced by Streptomyces ccelicolor, one such key binding event is between an ACP carrying a 16-carbon octaketide chain (act $\mathrm{ACP}$ ) and a ketoreductase (actKR). Once the octaketide is bound inside $a c t \mathrm{KR}$, it is likely cyclized between $\mathrm{C} 7$ and $\mathrm{C} 12$ and regioselective reduction of the ketone at $\mathrm{C} 9$ occurs: how these elegant chemical and conformational changes are controlled is not yet known. Here, we perform protein-protein docking, protein NMR, and extensive molecular dynamics simulations to reveal a probable mode of association between act ACP and actKR; we obtain and analyze a detailed model of the C7-C12-cyclized octaketide within the actKR active site; and confirm this model through multiscale (QM/MM) reaction simulations of the key ketoreduction step. Molecular dynamics simulations show that the most thermodynamically stable cyclized octaketide isomer $(7 S, 12 R)$ also gives rise to the most 'reactive conformations' for ketoreduction. Subsequent reaction simulations show that ketoreduction is stereoselective as well as regioselective, resulting in an $S$-alcohol. Our simulations further indicate several conserved residues that may be involved in selectivity of C7-12 cyclisation and C9 ketoreduction. The detailed insights obtained on ACP-based substrate presentation in type II PKSs will help with the design of nonendogenous ACP-ketoreductase systems capable of biosynthesizing non-natural polyketides.
\end{abstract}

\section{Introduction}

In type II polyketide synthases (PKS) ${ }^{1}$ a series of standalone enzymes grow a finite-sized polyketide chain and typically convert it into a complex natural product. An acyl carrier protein $(\mathrm{ACP})$ in conjunction with a chain-length factor (CLF) and ketosynthase (KS) heterodimer catalyze the basic carbon backbone assembly and define a minimal PKS complex. ${ }^{1-2}$ The polyketide alternates between being thioester linked to the KS or the phosphopantetheinyl (PPant) prosthetic group of the ACP (Chart 1) during each round of Claisen condensation of malonyl ACP and the KS bound polyketide. The ACP also shuttles the elongated substrate between subsequent tailoring PKS components that begin the defined series of chemical transformations that include reduction, cyclisation, aromatization, dimerization and numerous other functionalizations. ${ }^{2-3}$ The archetypal type II
PKS of the actinomycete bacterium Streptomyces colicolor $(\text { act } \mathrm{PKS})^{2-4}$ biosynthesizes the antibiotic actinorhodin (Chart 1) from a linear 16-carbon octaketide chain (1). After chain elongation, the actinorhodin ketoreductase (actKR) likely specifically cyclizes the polyketide between C7 and C12 (2) and reduces the ketone at $\mathrm{C} 9(3)$ while it remains attached to the actinorhodin ACP (act ACP) (Scheme 1). Extensive work by Tsai and co-workers has found the KR to be capable of remarkable regio- and stereocontrol ${ }^{5-8}$ and this class of enzymes has attracted interest for their use as biocatalysts in the stereoselective synthesis of small chiral alcohols from achiral ketones..$^{9-14}$ KRs can be mutated to alter and tune their biocatalytic properties as standalone enzymes or used in 'combinatorial biosynthesis' alongside other enzyme components from a PKS to produce polyketide derivatives with novel functionality. ${ }^{15-17}$ 
Chart 1. ActPKS octaketide and its products. ${ }^{a}$<smiles>Cc1cc(O)c2c(c1-c1cc(=O)cc(O)o1)C[C@@H](O)CC2=O</smiles><smiles>Cc1cc(O)c2c(c1O)C(=O)C1=C(C[C@H](CC(=O)O)O[C@@H]1C)C2=O</smiles>

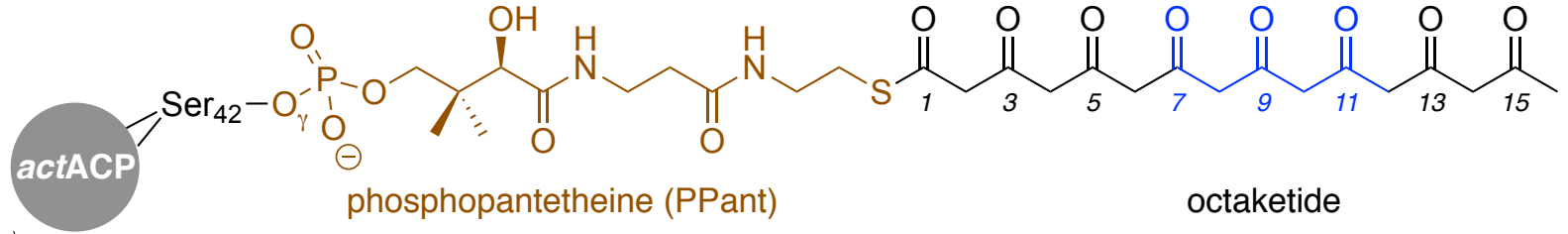

actACP-PPant-octaketide (actACP-1)

${ }^{a}$ Atoms in blue denote the portion of PPant-octaketide (1) that forms the six-membered ring upon cyclization

Scheme 1. Formation of cyclized octaketide 2 (A) and subsequent reactions (B). ${ }^{a}$

A

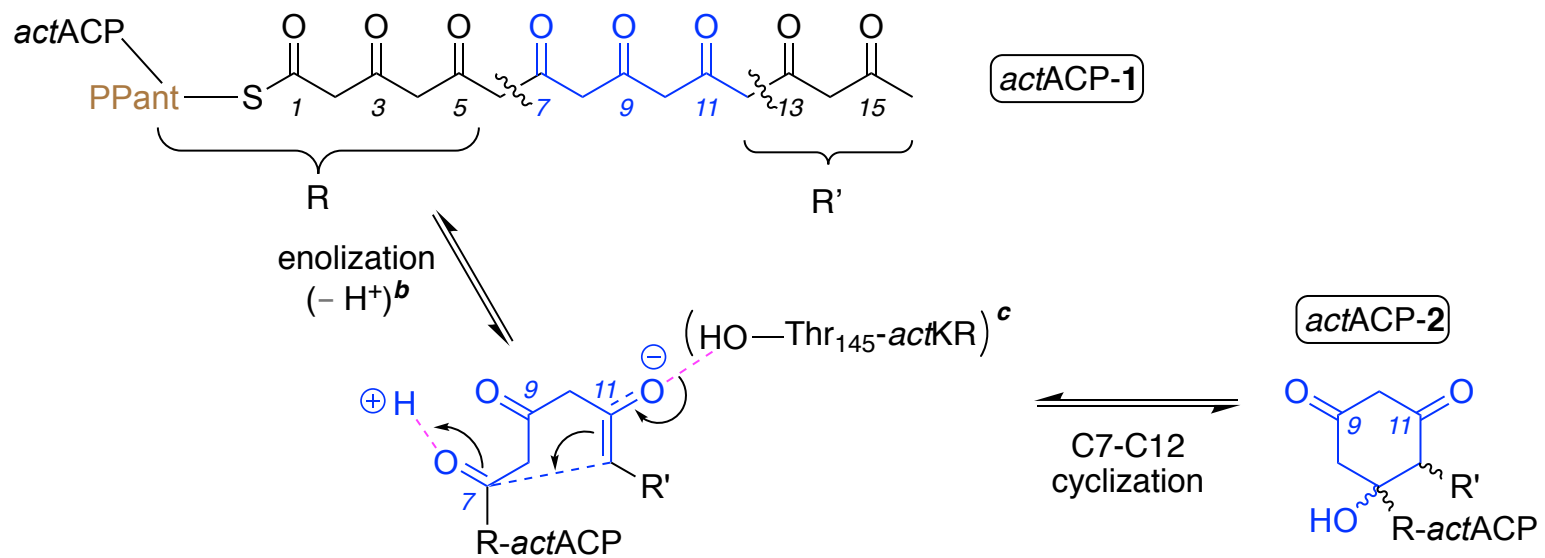

B

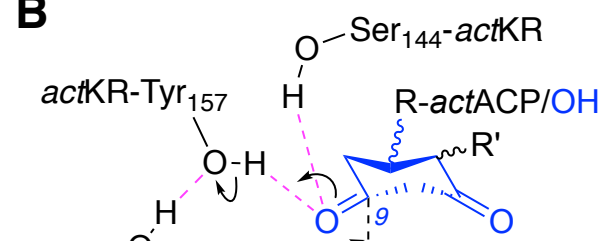

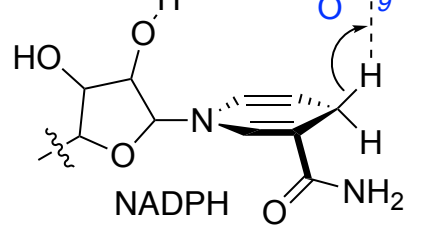

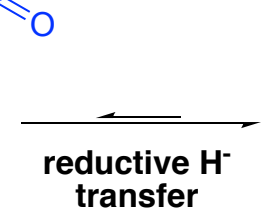<smiles>[R2]C1C(=O)CC(=O)C[C@]1(O)[R2]#N</smiles>

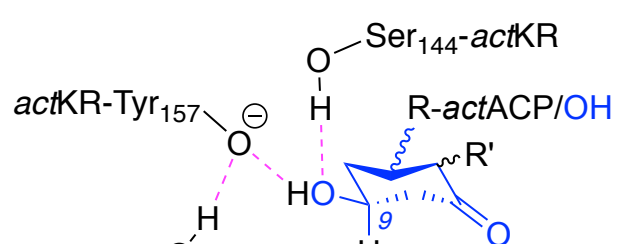

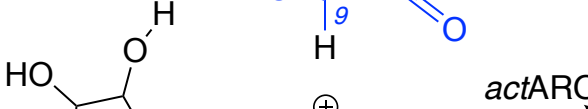<smiles>[R]c1c(O)cccc1[R20]#[Co]</smiles>

${ }^{a}$ Atoms in blue denote the portion of PPant-octaketide (1) forming the six-membered ring in the cyclized intermediate (2). Dotted magenta lines denote hydrogen bonds. ${ }^{b}$ Loss of a proton on C12. ${ }^{c}$ The exact role of actKR:Thr145 in cyclization is not established. 
Experimental and theoretical studies have confirmed that the PPant group, ${ }^{18}$ the $\alpha 2$ helix, ${ }^{19}$ and the $\alpha 3$ helix (acting as a conformational 'gatekeeper'; Figure $1 b)^{20}$ are all crucial in recognition of PKS and fatty acid synthase (FAS) ACPs ${ }^{21}$ by their enzymatic partners. ${ }^{22}$ In the actinorhodin system, the labile post-assembly octaketide substrate may be partially protected by act $\mathrm{ACP}^{23}$ whilst being transported to the actKR. ${ }^{1}$ The act $\mathrm{ACP}$ shuttle binds to one monomer of the homotetrameric actKR, whereupon $\mathbf{1}$ is unsheathed ${ }^{1}$ into its active site, which is characteristically narrow and restrictive. ${ }^{5}$ In the specific case of the actKR - act $\mathrm{ACP}$ interaction, it is known through mutagenesis studies that recognition is mediated by an 'arginine patch' formed by Arg38, Arg65, and $\operatorname{Arg} 93^{5,24}$ (Figure 1) that binds to the PPant phosphate. The pocket with the arginine patch further contains Asp109 and Thr113 (Figure 1c). ${ }^{5,8}$

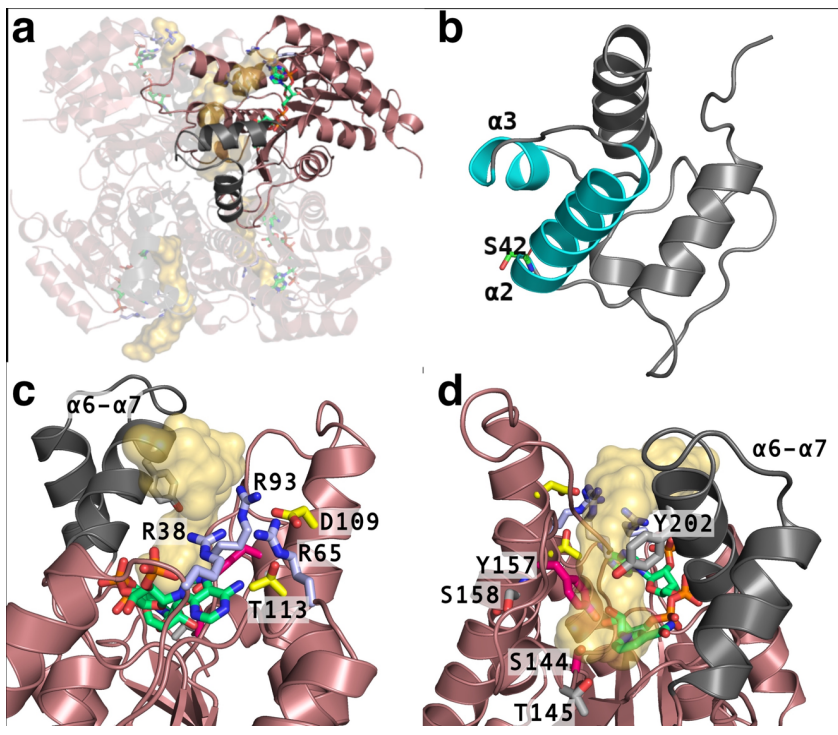

Figure 1. Features of $a c t \mathrm{KR}(\mathrm{PDB} 2 \mathrm{RH} 4)^{8}$ and $a c t \mathrm{ACP}$ (PDB $2 \mathrm{MVU})^{25}$ related to actKR-act ACP-substrate complex formation. (a) The actKR tetramer, with NADPH's $\mathrm{C}$ atoms in green, arginine patch (R38, R65, R93) in light blue and binding sites for the substrate as transparent orange surfaces highlighted in each monomer. (b) act ACP with the $\alpha 2$ and $\alpha 3$ helices in cyan and the PPant-bearing Ser42 highlighted. (c) actKR monomer (chain D) viewed from the side of the arginine patch, with D109 and T113 shown (C atoms in yellow). $\alpha 6-\alpha 7$ helices and loop are shown in dark gray. (d) As (c) with $180^{\circ}$ rotation highlighting putative catalytic residues for cyclization (1 to 2; T145, S158, and Y202; C atoms in light gray) and ketoreduction ( 2 to 3; S144, and Y157; C atoms in magenta).

The first transformation that likely takes place in actKR is the cyclization of actACP-1 (once spontaneously enolized) to yield actACP-2 (Scheme 1). ${ }^{5}$ The combination of ac$t \mathrm{ACP}-1$ binding to actKR monomers via the arginine patch ${ }^{6}$, ${ }^{8}$ and octaketide 1 docking inside the actKR's long but narrow active site ${ }^{5-6}$ probably allows the enzyme to exert strong regiocontrol that favours a $\mathrm{C} 7$ and $\mathrm{C} 12$ ring closure. $\mathrm{C} 7-\mathrm{C} 12$ cyclization is evident in the final product actinorhodin and the shunt product mutactin formed by the action of only the minimal PKS and actKR (Chart 1). In the absence of the act KR, C10-C15 cyclisation of $\mathbf{1}$ competes with the natural C7-C12 ring closure. ${ }^{5-6}$ Structure-activity relationships and sequence conservation led to Thr145 and Ser158 being proposed to play a role in this stereocontrol. ${ }^{5} \mathrm{Thr} 145$ has been suggested to play a role in stabilizing the $\mathrm{O}_{1} 1^{-}$enolate while Ser158 may assist in the proton transfer to O7 from the solvent.

The main (second) transformation in actKR - its ketoreduction of act ACP-2 - is both regio- and stereoselective, ${ }^{5}$ producing an alcohol group on C9 (Scheme 1). The first and rate-determining ${ }^{26}$ step in this reaction involves hydride transfer from the actKR-bound NADPH to $\mathrm{C}^{7,27}$ and (asynchronous concerted) proton abstraction by $\mathrm{O} 9$ from actKR:Tyr157. Stabilization is provided throughout the reaction by a hydrogen bond between $\mathrm{O} 9$ and actKR:Ser144 (Scheme 1). The chirality set by actKR at $\mathrm{C} 9$ remains unresolved; both $\mathbf{2}$ and $\mathbf{3}$ are too labile to be isolated. $\mathbf{3}$ is shuttled as actACP-3 to actinorhodin aromatase (actARO) and aromatized to act ACP-4, leading to a loss of the stereochemical information. In the absence of actARO, mutactin is generated, but its chirality has not been unambiguously confirmed; its designation as ' $9 S$ ' in Chart 1 is based on previous supposition. ${ }^{6}$

It has therefore not yet been possible to infer which stereoisomer and conformer of actACP-2, if any, is preferentially formed and reduced within actKR; nor how. Therefore, to fully understand the factors that connect the regio- and stereoselectivity, both the protein-protein and protein-substrate interactions between actKR and act ACP-1 should be considered in detail. Although binding models have been suggested, ${ }^{5,7,24}$ no crystal structure of the complex exists and detail on the interactions between act $\mathrm{ACP}$ and $\operatorname{act} \mathrm{KR}$ is lacking. Most solved enzyme-ACP complexes ${ }^{28-40}$ feature FASs,${ }^{28-34}$ and only two feature a PKS component (namely, ketosynthase). ${ }^{35-36}$ Moreover, to study protein-substrate interactions, a complex with act ACP-1 is required, but this is unfeasible. In this work, we combine protein-protein docking, molecular dynamics (MD) simulations, 2D proteinNMR spectroscopy and hybrid quantum mechanics / molecular mechanics (QM/MM) simulations to obtain detailed information on act $\mathrm{KR}$ - act $\mathrm{ACP}$ binding and act $\mathrm{KR}$ - octaketide interactions: our aim is to provide a unified picture of actKR structure and function and address the lack of fundamental knowledge on type II PKSs. ${ }^{1}, 17,22$ 


\section{Materials and Methods}

Protein-protein Docking. Rigid docking calculations of actKR-NADPH and apo actACP were performed using the Bristol University Docking Engine $(B U D E),{ }^{41}$ with GPU acceleration. The structure for actKR-NADPH was obtained from previous simulations ${ }^{27}$ starting from PDB ID 2RH4, ${ }^{8}$ and the structure of actACP was taken from model 13 of the NMR ensemble PDB ID 2MVU ${ }^{25}$ (see details in Supporting Information). To maximize docking efficiency, the search space was restricted to areas of each protein's accessible surface interfaces and excluded areas too far away from the arginine patch. For docking, a "generation zero" of 4600 poses was randomly generated for each of the 43625 possible pairs of chosen actKR-NADPH and act ACP surface points. The 50 highest-scoring poses were evolved into 2500 "generation-one children" using a Monte Carlo algorithm, and the process (50 new parents, 2500 new children) repeated to generation five, resulting in $\sim 3000$ fifth-generation binding modes. 17 binding modes (labeled M4-M20) were selected (based on BUDE score and the distance of actACP:Ser42 to the arginine patch) and for comparison, three actKR-ac$t$ ACP models obtained or derived from previous works ${ }^{5,24,31}$ (M1-M3) were included. Detailed procedures and coordinates for all models are provided as Supporting Information.

MD simulations of actKR-act ACP complexes. Tetrameric (actKR-NADPH) 4 -(actACP)4 structures for molecular mechanical (MM) MD simulations were assembled from the docking results, initially using different docking models (M1-M20) at each actKR chain in the tetramer, without the PPant-octaketide (see Figure 2, series $\mathrm{I}_{\mathrm{A}}$ ). For three docking models that gave the most promising results in series $\mathrm{I}_{\mathrm{A}}$ (M10, M14 and M17), further simulations were run with four act ACPs from the same model bound to one actKR tetramer (see Figure 2, series $I_{B}$ ). For the model selected after NMR assessment (M14), further MD simulations of the tetrameric complex were performed after introducing the PPant-octaketide moiety (2), with all combinations of the possible cyclization conformers (stage II, Figure 2; Scheme 2; Table S3). 2 was modelled from different starting positions in the active sites of each system by finding a balance between: 1) conformational agreement with the PPant of octaketide mimics crystallized with $\mathrm{KRs} ;{ }^{42-44}$ and 2) maintaining catalytic interactions with residues Ser144 and Tyr157. The latter was not possible with $C 9$ positioned for pro- $R$ hydride attack; starting structures therefore had $S$ prochirality in all cases. ${ }^{6}$ Compared to the MD simulations without PPant-octaketide, the $\alpha 6-\alpha 7$ loops of actKR and adjacent residues (188-229) were positioned in a more 'closed' form, with the Tyr202 side chain projecting inside the active site (and a water molecule bridging Tyr202 and the octaketide). ${ }^{44}$

For both stage I and II MD, all residues were in their standard protonation states (consistent with $\mathrm{p} K_{\mathrm{a}}$ predictions from PROPKA 3.1) $)^{45}$ with actKR His 162 protonated on $\mathrm{N} \delta 1$ and His 153 and His201 on Ne2 (according to the surrounding Hbond network). All systems were solvated in a rectangular box extending at least $11 \AA$ from any protein atom and neutralized by addition of $\mathrm{Na}^{+}$ions. The ff1 $4 S B$ force field ${ }^{46}$ and the TIP3P model $^{47}$ were used, alongside NADPH parameters from Holmberg and coworkers ${ }^{48}$. GAFF ${ }^{49}$ parameters with
HF/6-31(d) RESP point charges were used for the PPantSer42 fragment (details and libraries in SI; calculations in ioChem-BD). ${ }^{50-51}$ Multiple independent 32 ns periodicboundary MD runs were performed in the $N p T$ ensemble (after an equilibration procedure), using a 2 fs timestep (with SHAKE for bonds containing hydrogen). The temperature was maintained at $303 \mathrm{~K}$, in line with kinetic assays ${ }^{8}$ and recommended assessment of protein-protein docking stability, ${ }^{52}$ and pressure at $1 \mathrm{~atm}$. All simulations are conducted using AMBER $16^{53-54}$ with GPU acceleration where applicable. CPPTRAJ ${ }^{55}$ is used for trajectory analysis and postprocessing. Further details on generation of starting structures and MD procedures are provided in Supporting Information.

QM/MM reaction simulation of ketoreduction. $\mathrm{QM} / \mathrm{MM}$ MD Umbrella Sampling (US) reaction simulations were run with sander from AMBER 16. ${ }^{56-57}$ Simulation conditions were identical to the MM MD production runs, except for a shorter time-step (1 vs. 2 fs) and no SHAKE restraints ${ }^{58}$ on the $\mathrm{QM}$ region. This region was limited to one active site, and comprised the cyclooctaketide moiety of $\mathbf{2}$ from $\mathrm{C} 4$ to $\mathrm{C} 16$; Ser144 and Tyr157 side chains from C $\beta$; the nicotinamide moiety of NADPH up to the first ribose (Figure S2). The QM region was treated with the semiempirical method $\mathrm{PM}^{59}$ as used and benchmarked in our previous study on actKR (PM6 overestimates the barrier, but the mechanism is correct). ${ }^{27} \mathrm{QM} / \mathrm{MM}$ MD US simulations of reductive hydride transfer from NADPH to 2's C9 were run as previously $^{27}$, using the difference $(x-y)$ as reaction coordinate, where $y$ is the distance NADPH: $\mathrm{H}^{-}-2: \mathrm{C} 9$ and $x$ is the distance NADPH: $\mathrm{H}^{-}-\mathrm{NADPH}: \mathrm{C}_{\mathrm{H}}-$ (Figure S2). Simulations were started from 11 or 12 different 'reactive conformations' selected from stage II MD runs for each of the three isomers of 2 for which reactive conformations were regularly sampled (vide infra). The reaction coordinate was followed using US windows $0.1 \AA$ apart until reaching $1.8 \AA$, and free energy profiles were obtained by combining all sampling ( $\sim 1$ ns per isomer) using the weighted histogram analysis method. ${ }^{60-61}$ Further details are reported in the Supporting Information.

2D-NMR titration of actKR into act ACP. act $\mathrm{KR}^{5}$ and uniformly ${ }^{15} \mathrm{~N}$ labelled act $\mathrm{ACP}^{25}$ were expressed and purified as described previously. All NMR data was acquired with a Varian INOVA $600 \mathrm{MHz}$ spectrometer at $25^{\circ} \mathrm{C}$. Titrations of actKR into ${ }^{15} \mathrm{~N}$-labelled holo-act ACP were monitored by ${ }^{1} \mathrm{H}-{ }^{15} \mathrm{~N}$ HSQC-TROSY experiments. The molar ratios of $\mathrm{KR}: \mathrm{ACP}$ at each titration point were $0.08,0.47,0.33,0.67$, $1.00,1.34,1.67$ and 2.34 respectively. Stock solution of KR was $1.66 \mathrm{mM} \mathrm{KR}$ in $100 \mathrm{mM}$ potassium phosphate $\mathrm{pH}$ 6.5, $10 \mathrm{mM}$ EDTA and $1 \mathrm{mM}$ DTT. This was added to $500 \mu \mathrm{l}$ of $0.5 \mathrm{mM}^{15} \mathrm{~N}$-labelled ACP in the same buffer. 


\section{Results and Discussion}

Approach for actKR-actACP model generation and validation. To obtain and validate a reliable, detailed structural model for act ACP-2 binding to the tetrameric actKR, a stepwise computational procedure was followed (Figure 2), guided by NMR spectroscopy. The general approach is in line with recent recommendations ${ }^{1,62}$ and previous work on related systems. ${ }^{63-64}$ First, protein-protein docking was used to explore potential actKR - act ACP binding modes. Selected modes were then refined ${ }^{52}$ through extensive classical molecular dynamics simulations, in absence of the PPantsubstrate (Figure 2; Stage I). Structural analysis based on chemical shift perturbations of actACP obtained from 2D ${ }^{1} \mathrm{H}_{-}{ }^{15} \mathrm{~N}$ HSQC actKR titration data helped select the most likely binding mode. Then, all four stereoisomers of $\mathbf{2}$ were modelled into this binding mode, using all 8 possible cyclized species, referred to as 'isomer-conformers' (see Scheme 2 below). Thereafter, detailed molecular mechanical and hybrid QM/MM molecular dynamics simulations test the enzyme-substrate interactions and expected reactivity (final two stages in Figure 2). In the following subsections, we describe results from each stage in more detail and discuss how the validated model informs on the origins of stereo- and regio-selectivity of actKR-act ACP.

ActKR-actACP Binding Poses from Docking and MD simulation. Previous work ${ }^{5,8}$ has indicated that the act ACP act $\mathrm{KR}$ interaction is guided by a patch of three arginines on actKR (Figure 1), which recognize and bind the PPant phosphate attached to Ser42 in actACP-1. ${ }^{5,24}$ Extensive proteinprotein docking of an actKR monomer (with NADPH bound) and actACP (in the absence of substrate) was assessed (Protein-protein Docking in Figure 2) alongside two previously suggested binding modes (M1 ${ }^{24}$ and M2), ${ }^{5}$ and one (M3) derived from the crystal structure of Escherichia coli enoyl reductase FabI complexed to its ACP (PDB 2FHS). ${ }^{31}$ By considering a combination of the interaction energy (BUDE docking score) and a cutoff for the distance ( $d$ (PPant-actKR)) between act ACP:Ser42:C $\beta$ (bound to the $\mathrm{O} \gamma$ which carries the PPant) and actKR:Arg38:C (representing the arginine patch), henceforth referred to as Ser42patch distance, we selected 17 further docking models (M4M20) that are structurally distinct (see Supporting Information).

The full set of models M1-M20 were then further assessed using classical molecular dynamics (MD; MD Stage I in Figure 2) simulations to compensate for the rigidity in docking and to help eliminate false positives. ${ }^{52}$ For each model, 8 MD-refined binding poses were obtained through 8 independent MD simulations of $32 \mathrm{~ns}$ and subsequent clustering (series $\mathbf{I}_{\mathbf{A}}$ in Figure 2, details in Supporting Information). For each MD refined binding pose ( 8 for each of the 20 docking models, i.e., 160 in total), interaction energy (BUDE score) and $d$ (PPant-actKR) were measured (Figure S4). Binding modes M1-M3, M5-8, M11, M12 and M19 were deemed non-viable after MD refinement: all poses from $\mathbf{M 2}$ and $\mathbf{M 3}$ had large Ser42-patch distances ( $>24 \AA)$ and unfavorable interaction energies $\left(>-90 \mathrm{~kJ} \mathrm{~mol}^{-1}\right)$; all poses from M1,M5-8 had Ser42-patch distances $>9 \AA$ (even though 24 poses out of 40 had interaction energies between -174 and $-90 \mathrm{~kJ} \mathrm{~mol}^{-}$
${ }^{1}$ ); all poses from M11, M12 and M19 had consistently poor interaction energies $\left(>-90 \mathrm{~kJ} \mathrm{~mol}^{-1}\right)$.

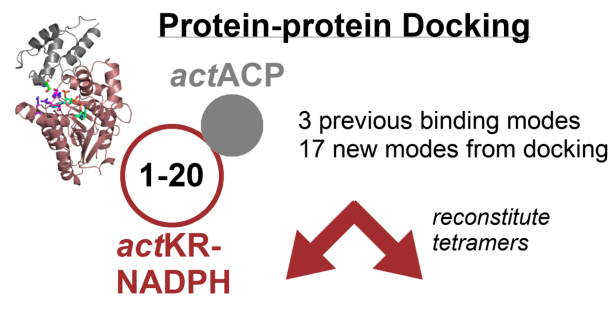

MD Stage I (Tetrameric Complex)
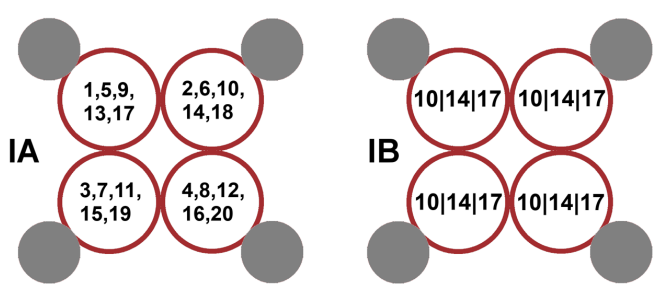

IA: 4 different binding modes per tetramer 8 independent simulations per tetramer All 20 modes covered ( 5 tetramers)

IB: 4 copies of same binding mode per tetramer 4 independent simulations per tetramer Binding modes M10, M14 or M17 (3 tetramers)

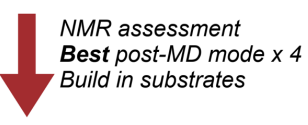

\section{Stage II (Complex + Substrate)}

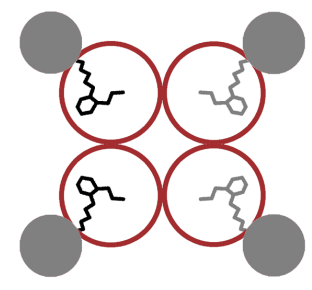

$16 \operatorname{sim} . R R-2+R S-2$ (8 OHax $+8 \mathrm{OHeq})$

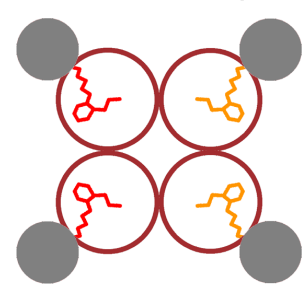

16 sim. SR-2 + SS-2 $(8 \mathrm{OHax}+8 \mathrm{OHeq})$

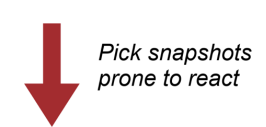

QM/MM reaction simulations
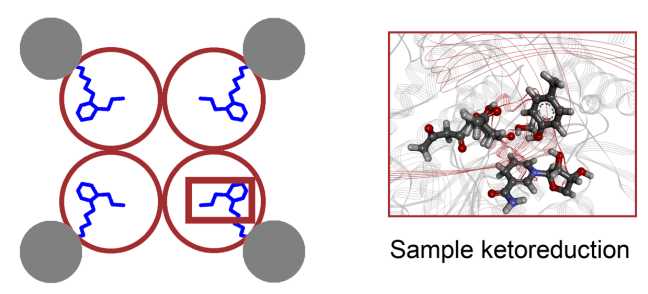

Sample ketoreduction

Figure 2. Overview of the computational procedure for actACPactKR model generation and validation. Protein-protein docking is followed by MD simulations in the absence of $\mathbf{2}$. After assessment using act ACP-actKR NMR titration data, MD simulations in the presence of $\mathbf{2}$ and hybrid QM/MM reaction simulations are performed.

MD refinement of the remaining docking models (M4, M9, M10, M13-M18 and M20) yielded several examples with good protein-protein interaction and compatibility with PPant insertion (low BUDE score and Ser42-patch distance, 
$d(\mathrm{PPant}-a c t \mathrm{KR}))$. When applying thresholds of interaction energy below $-90 \mathrm{~kJ} \mathrm{~mol}^{-1}$ and Ser42-patch distance below $9 \AA$ (pink rectangle in Figure 3), there was one favorable binding pose each originating from docking models M4, M9, M15, M16, and M20 (Figure S4); two originating from M13 (Figure S4); three from M18 (Figure S4); and as many as four from M10 and M14 and five from M17 (Figure S4; triangles in Figure 3). All but two of these 23 refined poses improved their interaction energy from docking, indicating that the flexibility introduced by MD simulation generally improved the actKR-act ACP binding interface.

Based on the positive initial assessment (4 or 5 representative snapshots within the thresholds), docking models M10, M14 and M17 were selected for further MD simulation (Series $\mathbf{I}_{\mathbf{B}}$; note that the next-best model $\mathbf{M 1 8}$ is structurally similar to M14, with a RMSD between act ACP:C $\alpha$ atoms of only $1.59 \AA$, and was therefore not selected). MD simulations were then performed using only one binding mode at each act $\mathrm{KR}$-act $\mathrm{ACP}$ interface (using $4 \times 32$ ns simulations for each tetramer, Figure 2). Clustering then gave 16 additional representative snapshots for each binding mode. Using the same thresholds as before $\left(B U D E\right.$ score $<-90 \mathrm{~kJ} \mathrm{~mol}^{-1}$; $d($ PPant-actKR $)<9 \AA), 13$ additional binding poses were found for M10; 9 for M14, and just two for M17 (Figure 3, Figure S5). (All but one of the additional poses improved their interaction energy from docking.) To further assist in the selection of the most promising binding poses, we also considered whether the Ser42-patch distance remained within $15 \%$ of its original value during the last 4 ns of the MD simulation from which each pose was selected (Figure 3; filled-in symbols). Combining this criterion with the best interaction energy (first) and the shortest Ser42-patch distance (second) resulted in the selection of refined poses

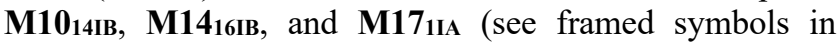

Figure 3 and structures in Figure 4). The subscripts denote the MD replica (number 14, 16, or 1) and series (IA or IB).

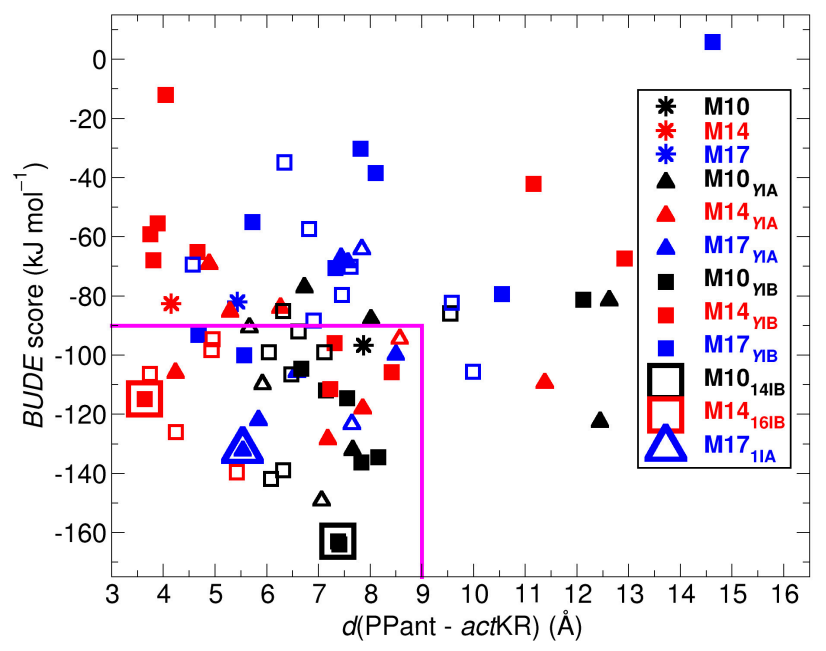

Figure 3. Refinement and ranking of docking models by MD simulation. ActKR-act ACP binding modes originating from MD simulations of M10 (black); M14 (red); and M17 (blue) are shown with their interaction energy (BUDE score, $y$-axis) and Ser42patch distance ( $d(\mathrm{PPant}-a c t \mathrm{KR}), x$-axis). Asterisks denote original docking modes; squares, those originating from series IA ( $\mathbf{M} \boldsymbol{X}_{\mathbf{Y I A}}$;

$X=10,14,17 ; Y=1-8)$; triangles, those originating from IB $\left(\mathbf{M} \boldsymbol{X}_{\mathbf{Y I B}} ; Y=1-16\right)$. The area bound by magenta lines indicates the region with BUDE score $<-90 \mathrm{~kJ} \mathrm{~mol}^{-1}$ and $\mathrm{d}($ PPant-actKR $)<9$ $\AA$ thresholds. Open triangles and squares refer to binding modes whose Ser42-patch distance deviates by more than $15 \%$ from its average value in the last $4 \mathrm{~ns}$ of the MD simulations from which they originate. The three binding modes selected for structural analysis and validation with NMR are highlighted by framed symbols. 

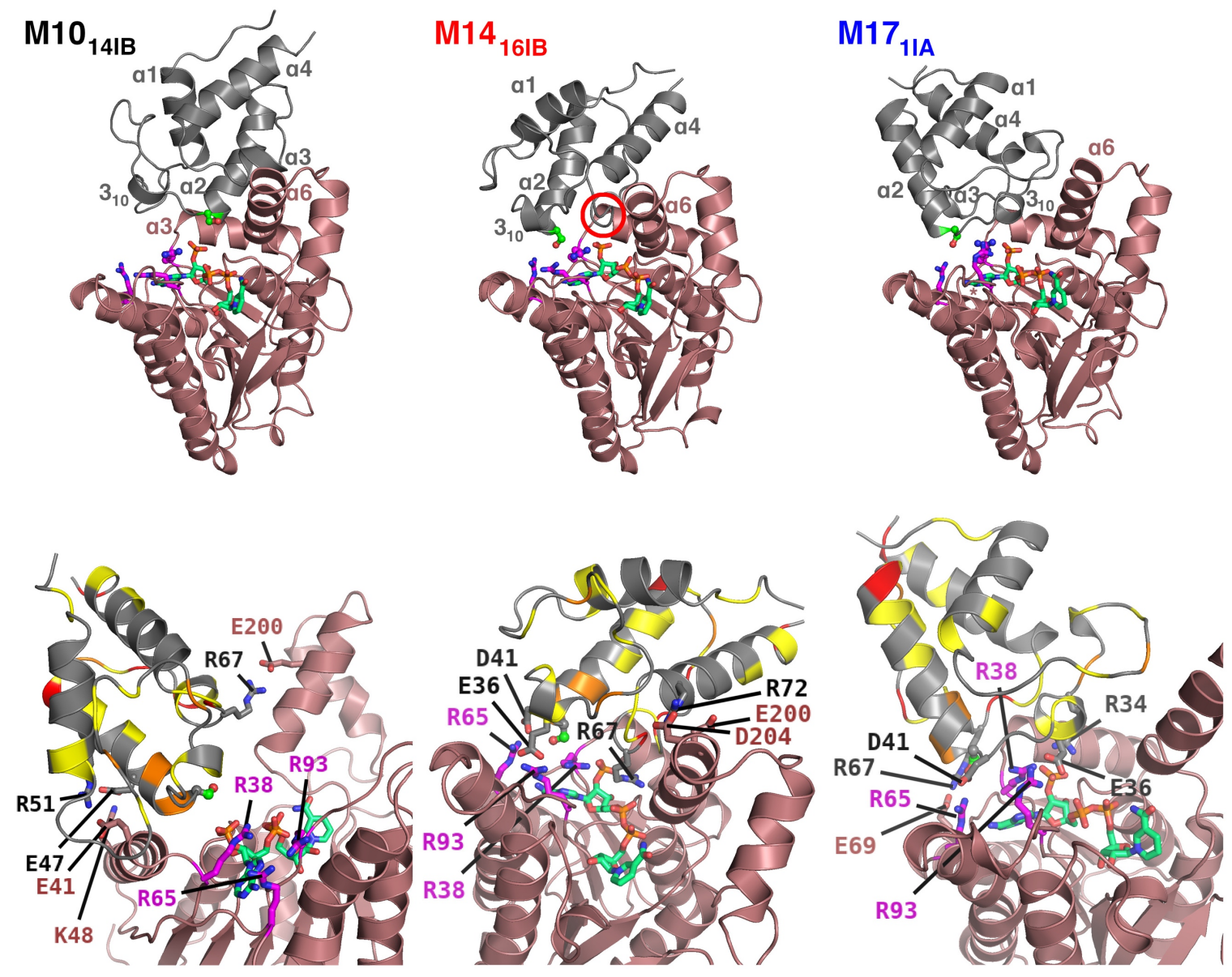

Figure 4. Putative act ACP-actKR binding modes M10 $_{14 \mathrm{IB}}$, M14 $_{16 \mathrm{IB}}$, and M17 $_{1 \mathrm{IA}}$ selected from docking and MD simulations. Top 3 panels: overview of act $\mathrm{ACP}-a c t \mathrm{KR}$ binding modes, with actKR (off-red cartoon) in the same orientation; act $\mathrm{ACP}$ as gray cartoon. In M14 $\mathbf{1 6 I B}$, act ACP's 'gatekeeper' $\alpha 3$ helix is marked by a red circle; it is central to the act ACP-actKR interface and has lost some of its structure. NADPH, actACP:Ser42, and the actKR arginine patch are shown (according to key below). Bottom 3 panels: magnification of the act ACPactKR interfaces with the ACP backbone colored according to the magnitude of the measured chemical shift perturbations upon addition of actKR: $0.02<\Delta \delta_{\mathrm{AV}}<0.04 \mathrm{ppm}$ in yellow, $0.04<\Delta \delta_{\mathrm{AV}}<0.06 \mathrm{ppm}$ in orange and $\Delta \delta_{\mathrm{AV}} \geqslant 0.06$ ppm in red (see Figure S6 for details). $\mathrm{NADPH}$, act ACP:Ser42, the actKR arginine patch and actKR and act ACP residues implicated in salt bridges (Table S5) are labelled and rendered as sticks. Key for all panels: NADPH with C atoms in green; Arginine patch with C atoms in magenta; actACP:Ser42 in ball-andstick with bright green $\mathrm{C}$ atoms; $\mathrm{H}$ atoms omitted for clarity.

Structural Analysis and 2D-NMR Validation of act ACPactKR Binding Modes. All three actKR-actACP binding modes selected after docking and MD simulation (Figure 4) feature actACP:Ser42 (at the $N$-terminus of actACP's $\alpha 2$ helix) relatively close to Arg38. Only in M14 16IB $_{\text {a }}$ and M17 ${ }_{1 I A}$, however, are all three arginines in the patch ${ }^{5,8}$ positioned to capture the phosphate in the PPant moiety of 2 (Figure 4): act ACP:Ser42:O $\gamma$ is 3.4 and $5.2 \AA$ away from center of mass of the arginine guanidinium moieties, respectively (vs. 12.9 $\AA$ away in M10 14IB ). The occluded surface area is larger in M14 $_{16 \text { IB }}\left(1747 \AA^{2}\right)$ than in M17 IIA $_{\left(1402 \AA^{2}\right) \text { and M10 }}$ 14IB $\left(1148 \AA^{2}\right)$, indicating a better fit between act $\mathrm{ACP}$ and actKR in the former. All three binding modes exhibit several stable salt bridge interactions between act ACP and actKR (Table S5). In M10 $14 \mathrm{IB}$, however, no salt bridges are formed with the arginine patch ${ }^{5,24}$ or NADPH (with most contacts between the act ACP $\alpha 2$ and actKR $\alpha 6$ helices). In contrast, in

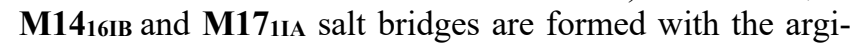
nine patch by both actACP:Asp41 and actACP:Glu36, and with the phosphate moieties of NADPH by Arg67 (M14 16IB) $_{1}$ or Arg34 (M17 1 IIA). In M14 $16 \mathrm{BB}$, actACP $\alpha 3$ is in the center of the act $\mathrm{ACP}-a c t \mathrm{KR}$ interface, whereas the overall binding interaction in M17 IIA $_{\text {in }}$ is dominated by the $\alpha 1-\alpha 2$ loop and does not involve $\alpha 3$.

To better distinguish the binding modes, we conducted ${ }^{1} \mathrm{H}-$ ${ }^{15} \mathrm{~N}$ HSQC titration experiments using ${ }^{15} \mathrm{~N}$ labeled act ACP and unlabelled actKR (Supporting Information; Figure S6). Titration to an excess of actKR:actACP showed distinct chemical shift perturbations (CSPs) particularly across the $\alpha 2-\alpha 3$ loop and $\alpha 3$. The largest magnitude CSPs are observed in this region (I60, D62, V68) and may also report on conformational changes in $\alpha 3$ as reported previously, ${ }^{20}$ again pointing to the involvement of $\alpha 3$ in the act $\mathrm{ACP}-$ act KR interaction, as observed in M14 1 Iв. Further, residues of the flexible $\alpha 1-\alpha 2$ loop from T21-D29 exhibited exchange broadening; this loop is fully solvent-exposed only in M14 1 16IB.

In summary, structural analysis and NMR titration suggests that binding mode M14 1 íB is most representative of the 
act $\mathrm{ACP}-a c t \mathrm{KR}$ complex, with the following features: (1) the act ACP 'gatekeeper' helix $(\alpha 3)$ is central to the interface, occupying a cleft above the central NADPH phosphates; (2) the $\alpha 4$ helix interacts with the (mobile) $\alpha 6$ helix of actKR; (3) part of the $\alpha 2-\alpha 3$ loop, indicated by Hadfield et al. as being important for protein-protein interactions ${ }^{24}$ is also in contact with actKR; and (4) the $\alpha 1-\alpha 2$ loop is solvent exposed.

Reactivity of ACP-bound Cyclized Octaketides in actKR. To assess the possible binding interactions of cyclized octaketides in actKR, we performed multiple independent MD simulations of act KR - act ACP with all possible cyclized conformers of 2 (MD stage II in Figure 2): C7-C12 cyclization of 1 can, in principle, lead to four different stereoisomers of 2 (color-coded in Scheme 2): (7R,12R)-2 (henceforth $R R$-2; black); (7R,12S)-2 (RS-2; gray); (7S, 12R)-2 (SR2; red); and (7S, 12S)-2 (SS-2; orange). In turn, each of these four isomers can access two low-energy chair conformers (Scheme 2), with the $\mathrm{C} 7-\mathrm{OH}$ substituent oriented either axially $\left(\mathbf{2}_{\mathrm{OHax}}\right)$ or equatorially $\left(\mathbf{2}_{\mathrm{OHeq}}\right)$. act ACP binding mode M14 ${ }_{16 \text { IB }}$ was used and the $a c t K R ~ \alpha 6-\alpha 7$ loop was remodeled prior to MD simulation, based on an actKR-octaketide mimic complex structure, ${ }^{44}$ in line with the suggested role of this loop in substrate recognition. ${ }^{5}$ By using previous structural information ${ }^{42-44}$ and satisfying contacts between $\mathbf{2}$ and catalytic residues, initial placements for the PPant and the octaketide moieties were generated (two alternative starting positions were used in order to explore a greater portion of conformational space; modelling details and coordinates are included as Supporting Information).

In the resulting MD simulations, the frequency of reactive poses (\%reac, defined by satisfying key distances; see Supporting Information) ${ }^{65}$ of 2 towards C9 ketoreduction was monitored, and the combined \% reac values (from 4 active sites $\times 8$ replicas $\times 32 \mathrm{~ns} \times$ four initial systems, see Table S3) were compared for each of the eight possible cyclization isomer-conformers of $\mathbf{2}$ (Figure 5, Table S6). SR-2 ${ }_{\mathrm{OHax}}$ had the highest frequency of reactive poses (at 9.1\%), followed by $R R-2_{\mathrm{OHeq}}$ and $R S-\mathbf{2}_{\mathrm{OHeq}}$ (with $2.9 \%$ and $2.2 \%$, respectively). The remaining five isomer-conformers had less than $2 \%$ reactive poses. Essentially all reactive poses are pro-S; only 12 pro- $R$ poses were observed for all isomer-conformers (all for $S S-2_{\mathrm{OHeq}}$ ) out of a total of $>1.5$ million snapshots. Our simulations thus show that $\mathbf{2}$ is (much) more prone to pro-S hydride attack at $\mathrm{C}$, in agreement with previous in silico models of the presentation of the polyketide substrate to $\mathrm{NADPH}^{6}$ (see further below).

We further considered the relative free energies of all eight isomer-conformers (Figure 5, $y$-axis; QM calculation details in Supporting Information, optimized structures in ioChemBD). ${ }^{50-51}$ There is some degree of correlation between thermodynamic stability and the propensity to form reactive poses (e.g., Figure 6b) in the actKR active site: $S R-\mathbf{2}_{\mathrm{OHax}}$ is most stable, followed by $R S-\mathbf{2}_{\mathrm{OHeq}}$ and $R R-\mathbf{2}_{\mathrm{OHeq}}(0.9 \mathrm{kcal}$ $\mathrm{mol}^{-1}$ and $1.4 \mathrm{kcal} \mathrm{mol}^{-1}$ higher in energy, respectively). The remaining isomers are significantly higher in energy ( 2.5 to $5.6 \mathrm{kcal} \mathrm{mol}^{-1}$ ). There is also correlation between the frequency of reactive poses and stability of axial vs. equatorial $\mathrm{C} 7-\mathrm{OH}$ arrangement (especially for $12 \mathrm{R}$ isomers): in $7 \mathrm{~S}$ isomers, axial conformers are more stable and attain more reactive poses; in $7 R$ isomers, the opposite is true.

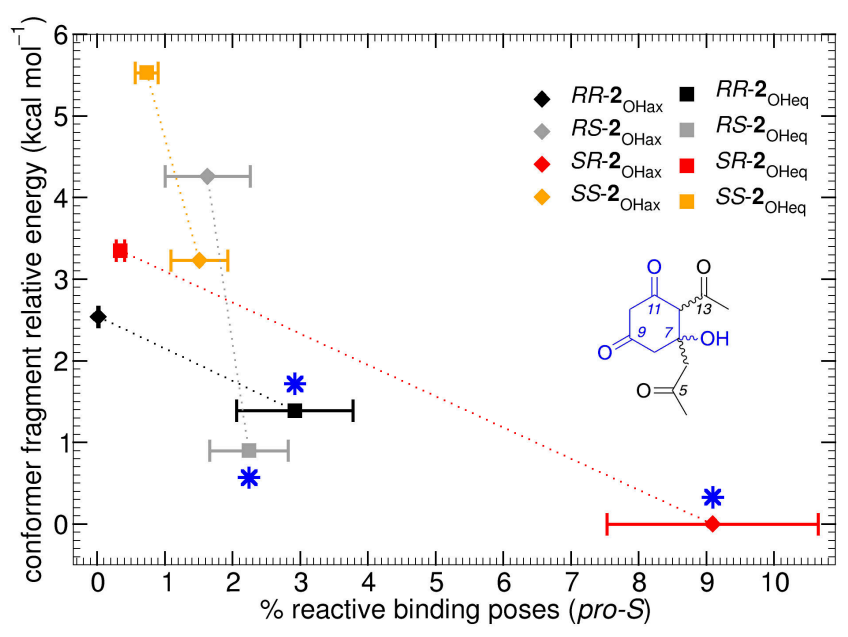

Figure 5. Comparison of QM energies and in-enzyme reactive poses of the different isomer-conformers of the cyclized octaketide 2. $x$-axis: percentage of pro-S reactive binding poses (\% $\%$ reac) present in stage II MD simulations (see Supporting Information for definition). $y$-axis: relative free energies of the C4-C14 fragment (SCS-MP2/6-31+G $(d, p) / / \mathrm{B} 3 \mathrm{LYP} / 6-31+\mathrm{G}(d, p)$, see Supporting Information for details) of the hydrogen-capped cyclopentaketide fragments (see inset). Lines are shown to guide the eye. $\left(^{*}\right)$ marks isomer-conformers chosen for QM/MM reaction simulations. Species are color-coded and labelled as in Scheme 2. Error bars along the $x$-axis are based on a leave-one-out procedure (see Supporting Information). 
Scheme 2. C7-C12 Cyclization of the Natural Substrate of actKR with its Possible Stereoisomers and Chair Conformers. ${ }^{a}$
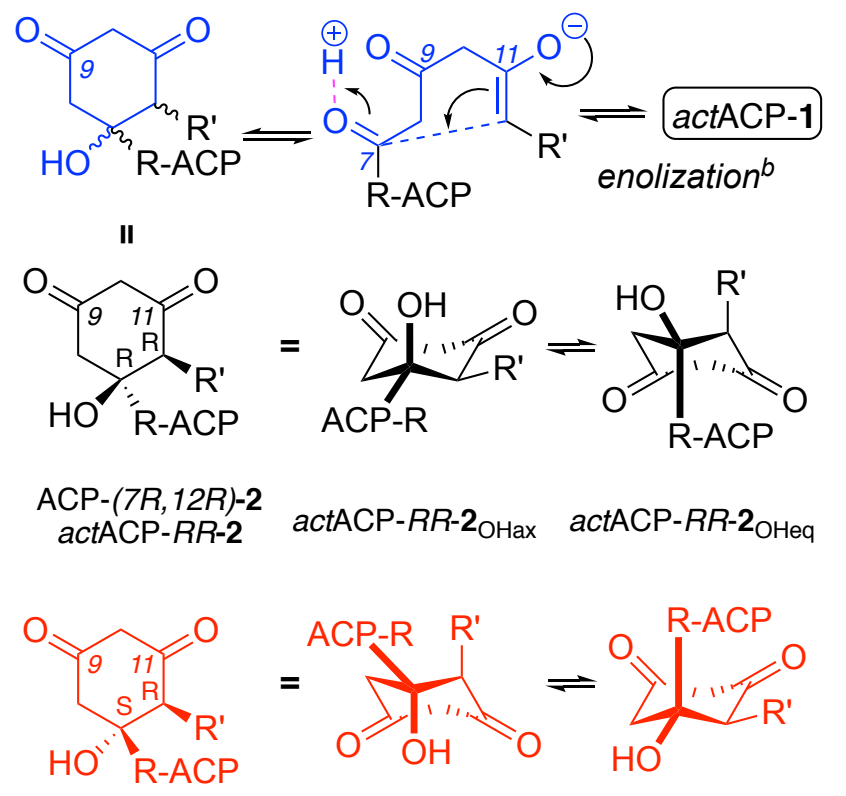

ACP- $(7 S, 12 R)-2$
actACP-SR-2 actACP-SR-2 ${ }_{\mathrm{OHax}}$ actACP-SR-2 $\mathbf{O H e q}_{\mathrm{H}}$

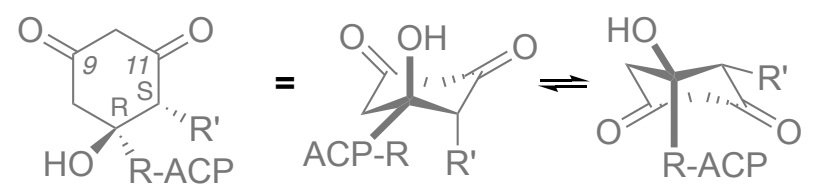

ACP-(7R, $12 S)-2$
actACP-RS-2 actACP-RS-2 ${ }_{\mathrm{OHax}}$ actACP-RS-2 $\mathbf{O H e q}_{\mathrm{H}}$

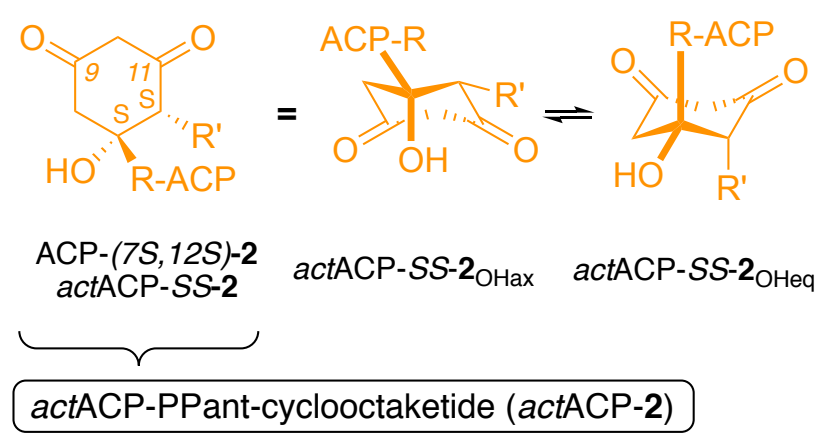

${ }^{a}$ Atoms in blue denote the portion of PPant-octaketide (1) forming the six-membered ring in the cyclized intermediate (2). ${ }^{b}$ Loss of a proton on $\mathrm{C} 12$.

To simulate the chemical reaction itself, we selected three isomer-conformers of $\mathbf{2}\left(S R-\mathbf{2}_{\mathrm{OHax}}, R S-\mathbf{2}_{\mathrm{OHeq}}\right.$, and $\left.R R-\mathbf{2}_{\mathrm{OHeq}}\right)$. As indicated by the relatively infrequent occurrence of reactive poses (at most $9.1 \%$ for $S R-2_{\mathrm{OHax}}$ ), the cyclized octaketide spends the majority of the time 'in standby', i.e., bound in the active site with $\mathrm{C} 9$ close to the catalytic residues, but not quite ready for reaction (Figure 6a). Moving to a reactive conformation (e.g, Figure $6 \mathrm{~b}$ ) will thus come at a slight free energy cost $\left(1.4,2.1\right.$ or $2.3 \mathrm{kcal} \mathrm{mol}^{-1}$ at room temperature for $S R-\mathbf{2}_{\mathrm{OHax}}, R S-\mathbf{2}_{\mathrm{OHeq}}$, and $R R-\mathbf{2}_{\mathrm{OHeq}}$, respectively, based on $\Delta G=\mathrm{RT} \ln [\%$ reactive poses $]$ ). For each, we performed combined quantum mechanical / molecular mechanical (QM/MM) MD simulations of ketoreduction at $\mathrm{C}$ 9 (see $Q M / M M$ reaction simulations in Figure 2), using the same approach as our previous work on the reduction of trans-1-decalone by actKR. ${ }^{27}$ The transition states and reaction barriers obtained here are similar (Figure 6, Figure S3), which demonstrates that the selected complexes modeled

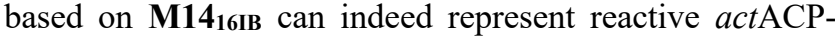
actKR poses, further validating this MD-refined model. As expected, the transition state corresponds to the hydride transfer between NADPH and C9, concerted with proton transfer from Tyr157 to O9 (Figure 6c). Subsequently, Tyr157 moves to coordinate to a NADP+ ribose hydroxyl (Figure 6d), ready for reprotonation through a proton shuttle likely involving the ribose and Lys $161 .{ }^{26-27}$ Notably, our simulations show energetically feasible reactions whilst the cyclized octaketide is bound to act $\mathrm{ACP}$, confirming that the ACP-PPant tether does not need to be broken prior to ketoreduction by actKR (in contrast to what is expected for hedamycin KR). ${ }^{9}$ The barriers to reaction are not significantly different between the three isomer-conformers, suggesting that $a c t \mathrm{KR}$ can facilitate ketoreduction to a similar extent in all three, via axial hydride attack at C9 (Scheme 1, Figure 6b-d). Whilst a preference for axial attack is in line with previous findings on the reduction of small alicyclic ketones by agents such as $\left[\mathrm{AlH}_{4}\right]^{-}$and $\left[\mathrm{BH}_{4}\right]^{-66-67}$ it is in contrast with findings by Østergaard et al. on reduction of the small alicyclic trans-1-decalone by another ketoreductase, ${ }^{68}$ and our own findings for its reduction by actKR itself. ${ }^{27}$ It appears that the tendency of actKR to catalyze equatorial $\mathrm{H}^{-}$ attack in small, non-endogenous substrates can be overridden by factors such as binding site architecture, spatial constraints arising from act KR-act $\mathrm{ACP}$ binding, and the presence of oxygen substituents on $\mathrm{C} 7$ and $\mathrm{C} 11$.

Determinants of actKR Stereo- and Regioselectivity. The near-absence of pro- $R$ reactive poses in our MD simulations (stage II) indicates that $S$-selectivity for ketoreduction at C9 in $a c t K R$ is defined by its active site structure in combination with the position of the incoming PPant chain, as suggested previously $^{5-6}$ (Figure 1d; Figure 6; Figure S2). The side chains of the adjacent residues actKR:Thr145 (possibly stabilizing O11 during cyclization of 1 to 2; Scheme 2) ${ }^{5}$ and actKR:Ser144 (stabilizing O9 during ketoreduction; Scheme 1) form a relatively rigid template close to the nicotinamide ring of NADPH. When $\mathrm{O} 11$ and $\mathrm{O} 9$ bind to these residues upon arrival of $\mathbf{1}$ into the active site, $\mathrm{C} 7-\mathrm{C} 12$ cyclization to any isomer-conformer of $\mathbf{2}$ creates spatial constraints that strongly favor reductive hydride attack in a pro-S pose (i.e., 'from below' in Figure 6b-d). 


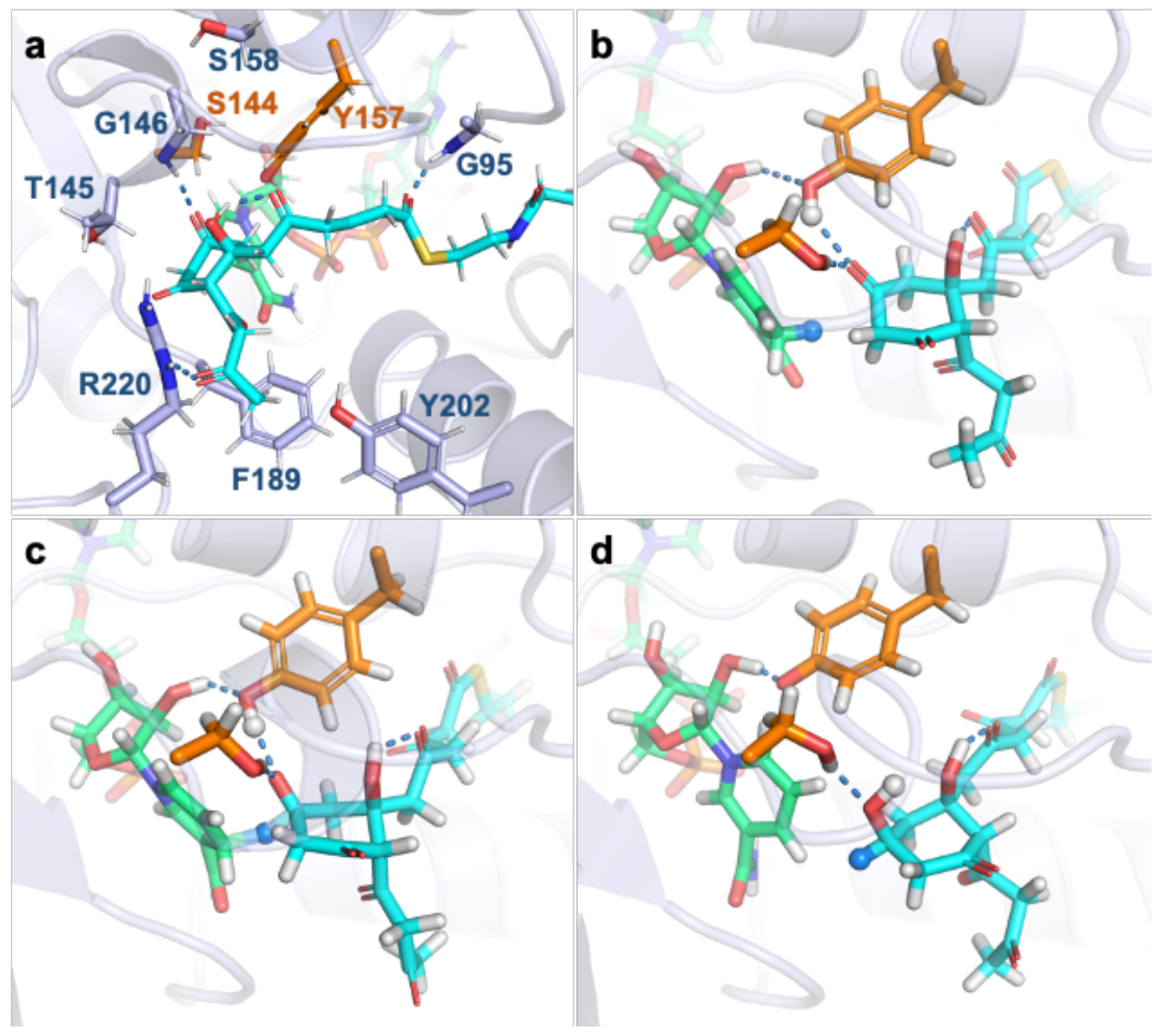

Figure 6. Key steps in the ketoreduction of actACP-SR-2 $\mathbf{O H a x}_{\text {by }}$ actKR. The sequence depicts the pro-S hydride attack on isomer-con-

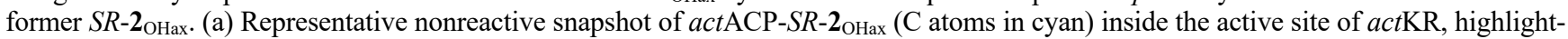
ing residues (sticks; $\mathrm{C}$ atoms light blue) that could be important for regioselectivity per our hydrogen bond analysis (see text). Catalytic residues Ser144, Tyr157 (C atoms in orange) and the NADPH cofactor (C atoms in green) are shown. Gly95:NH, part of the XGG motif, ${ }^{5-}$ 6,8 interacts frequently with 2:O1; other residues are discussed in the text. (b) Reactive pose of act ACP-SR-2 ${ }_{\mathrm{OHax}}$ poised for hydride transfer from NADPH. Hydride shown as blue sphere and actKR:Tyr157:H tion reaction, with hydride being transferred from NADPH to 2:C9. (d) Product of ketoreduction, with Tyr157's phenolic proton transferred to 2:09.

The link between $\mathrm{C} 7-\mathrm{C} 12$ ring conformer stability and greater propensity for $($ pro-S) $\mathrm{C} 9$ ketoreduction (i.e., the most energetically stable isomer-conformers also give the highest number of reactive poses) merits further reflection as to why the actKR active site might have evolved to harness isomer-conformers $S R-\mathbf{2}_{\mathrm{OHax}}, R S-\mathbf{2}_{\mathrm{OHeq}}$ and $R R-\mathbf{2}_{\text {OHeq }}$ in the first place. Perhaps the enzyme preferentially processes those conformers of $\mathbf{2}$ that are more likely to result after cyclization of $\mathbf{1}$. In addition, act ARO - likely having evolved in tandem with actKR - might prefer the combination of $S$ chirality at $\mathrm{C} 9$ alongside the three isomer-conformers to perform its conversion of $\mathbf{3}$ to $\mathbf{4}$ (although confirming this hypothesis would require detailed mechanistic studies of actARO, which is beyond the scope of this work).

Apart from its stereoselectivity in ketoreduction, the other remarkable characteristic of actKR is its regioselectivity: namely, why cyclization occurs between $\mathrm{C} 7$ and $\mathrm{C} 12$ (if it occurs on actKR, rather than on actKS/CLF), and why ketoreduction then occurs specifically at C9 (with the link between the two already noted). ${ }^{5}$ To investigate if and how the binding site architecture might drive regioselectivity, we examined the formation of hydrogen bonds (direct or watermediated) between actKR and substrate oxygen atoms in MD trajectories from stage II (Figure 6a, full details in Tables S7 and S8). Hydrogen bonds between the cyclized octaketide moiety and actKR are rather transient during our simulations. Short-lived hydrogen bonds are consistent with 1 and 3 'sliding' in and out of the binding channel, respectively, as suggested by Javidpour et al., ${ }^{5}$ as well as the 'in standby' conformation of the cyclized octaketide (with catalytically competent poses only being attained for a fraction of the simulation time, Figure 5). Hydrogen bonds directly relevant for ketoreduction at $\mathrm{C} 9$ are observed between $\mathrm{O} 9$ and Ser144 and Tyr157 on actKR (Scheme 1), but not as the most frequent (average frequencies, respectively, of 5.3 and $4.8 \%$ for $S R-\mathbf{2}, 4.3$ and $4.3 \%$ for $R R-\mathbf{2}$, and 3.0 and $1.8 \%$ for $R S$-2). Instead, the most frequent hydrogen bonding for $\mathrm{O} 9$ occurs with nearby backbone hydrogens of actKR:Phe189 (a residue whose importance was also noted experimentally) ${ }^{5,8}$ and actKR:Gly146 (Figure 6a, Table S7). Interactions with actKR:Ser144 and actKR:Tyr157's -OH hydrogens are typically mediated by water bridges when found 
(Table S8). These interactions are consistent with isomers of 2 being held 'in standby' in the binding site (Figure 6a), with the $\mathrm{C} 9=\mathrm{O} 9$ carbonyl never far from reaching a reactive pose (Figure 6b). Only this carbonyl interacts with the key catalytic residues, thus achieving regioselectivity at $\mathrm{C} 9$.

The simulated isomers of $\mathbf{2}$ can be considered as the products of C7-C12 cyclization of 1 (Scheme 2), and their interactions may therefore reflect how such regioselective cyclization might be promoted by the actKR active site. One possible key interaction could be hydrogen bonding between $\mathrm{O} 11$ and actKR:Thr145's hydroxyl group; however, our simulations only indicate sporadic and indirect hydrogen bond interactions (through water bridges, Table S8). A different hydrogen bond interaction that may be relevant for cyclization, between 2:07/H7 and actKR:Tyr202's -OH group, is observed occasionally in simulations for most isomer-conformer pairs (Table S7, Table S8). This (highly conserved) $)^{5}$ Tyr202 side chain, in its orientation towards the active site ${ }^{44}$ (Figure 6a, Figure S2), could thus be involved in catalyzing regioselective $\mathrm{C} 7-\mathrm{C} 12$ cyclization, e.g. as proton donor to $\mathrm{O} 7$, or aiding proton transfer from the nearby His153 and His201. Other interactions that may be relevant for cyclization are the long-lived intramolecular hydrogen bond between $2: \mathrm{H} 7$ and 2:O5, and the occasional water bridges between 2:O5 and actKR:Tyr202's -OH, both of which may contribute to C7-C12 cyclization through stabilization of proton transfer to O7. Notably, interactions of 2:O7/H7 with actKR:Ser158's hydroxyl group, previously proposed to play a role in proton donation in cyclization, ${ }^{5}$ are hardly ever sampled. While these specific hydrogen bond interactions detected for $\mathrm{O} 5, \mathrm{O} 7$, and $\mathrm{O} 11$ may be structurally and/or electronically important factors for regioselective cyclization of $\mathbf{1}$ to $\mathbf{2}$, further work is required to confirm the possible roles of actKR residues in C7-C12 cyclization, such as stabilization of the enolate species and the source for $\mathrm{O} 7$ protonation (e.g., involving Tyr202, His153 and/or His201).

Finally, we consider contacts at the extremities of the (cyclized) octaketide species. Zhao et al. recently used extensive MD simulations of actKR and a double mutant which affects chain-length specificity, together with octaketide and tetraketide substrate mimics. ${ }^{44}$ They considered two previously proposed substrate entrance sites, a 'back-patch' near Q149/R220 and a 'front-patch', identical to the 'arginine patch'. In our work, only binding at the latter is considered, as this is enforced by the location of ACP, with the PPant phosphate group binding to the arginine patch. This is consistent with the preference of the PPant octaketide mimic found by Zhao et al ${ }^{44}$ For the octaketide, we find frequent and fairly persistent hydrogen bonds (direct or through bridging waters) between the start of the chain $(\mathrm{O} 1)$ and the backbone actKR:Gly95:NH (Figure 6a). This glycine is part of the highly conserved XGG motif characterizing type II polyketide synthases, which has been suggested to be an anchor point for the PPant-octaketide to be presented to the actKR active site. ${ }^{5-6}$ Our simulations further support this. At the other end, $\mathrm{O} 15$ forms frequent hydrogen bonds (direct or through bridging waters) with actKR:Arg220, located towards the $C$-terminus of the $\alpha 7$ loop and previously considered by mutagenesis ${ }^{5}$ (Figure 6a). Arg220 can 'seal' the binding pocket at its far end (including through hydrogen bonding with actKR:Gln $149,{ }^{8}$ forming the 'back-patch' that can support binding of short polyketides) $)^{44}$ and could thus be a key factor for the regioselectivity of cyclization by helping the linear octaketide 1 buckle upon itself near O15, folding the $\mathrm{C} 12-\mathrm{C} 16$ fragment back onto $\mathrm{C} 7-\mathrm{C} 11$.

\section{Conclusions}

In the type II actinorhodin polyketide synthase, association between actinorhodin ketoreductase (actKR) and an actinorhodin acyl carrier protein (act ACP) carrying a phosphopantetheinylated octaketide results in the latter being inserted into the actKR active site (as $\mathbf{1}$ or cyclized as 2). Subsequently $\mathbf{2}$ is stereoselectively reduced at $\mathrm{C} 9=\mathrm{O} 9$ to yield alicyclic chiral alcohol 3. In this work, we have uncovered the most likely act $\mathrm{ACP}$ - actKR binding mode in atomic detail, using a combination of protein-protein docking, molecular dynamics simulations and NMR chemical shift perturbations. Then, further molecular dynamics simulations (including QM/MM reaction simulations) based on this binding mode are used investigate the mechanism and the sources of regio- and stereoselectivity of actKR towards its natural substrate.

After initial selection of simulation-refined docking models based on estimated binding affinity and proximity of $a c$ $t \mathrm{ACP}:$ Ser42 to a 'patch' of three arginines on actKR (Arg38, Arg65, Arg93), one binding mode was found to be most consistent with our 2D NMR data and previous reports. In this mode, act $\mathrm{ACP}$ docks onto actKR with its $\alpha 3$ helix and the $\mathrm{N}$-termini of $\alpha$-helices 2 and 4 . Subsequent simulations based on this binding mode of complexes with all possible C7-C12 cyclization isomers of 2 revealed an overwhelming preference for pro- $S$ ketoreduction at $\mathrm{C} 9=\mathrm{O} 9$, particularly for the most thermodynamically stable cyclization isomer (i.e., $(7 S, 12 R)-2$ with $\mathrm{C} 7-\mathrm{OH}$ oriented axially). As well as establishing a link between chirality at $\mathrm{C} 7 / \mathrm{C} 12$ and chirality at $\mathrm{C}$, this finding unequivocally confirms previous experimental data on mutactin inferring that $\mathrm{C} 9$ should be enantiopure; ${ }^{6}$ it also strongly suggests that chirality at 3:C9 should be $S$ rather than $R$, and that actKR preferentially catalyzes axial hydride attack at $\mathrm{C} 9$. The binding mode of act ACP in conjunction with spatial features of the actKR active site are sufficient to cause the indicated $S$-selectivity.

$\mathrm{QM} / \mathrm{MM} \mathrm{MD}$ reaction simulations of $\mathrm{C} 9$ ketoreduction were performed for the three isomers of $\mathbf{2}$ that most frequently formed reaction competent binding poses. This indicated that $S$-selective ketoreduction is equally efficient for these isomers (i.e., no specific $\mathrm{C} 7 / \mathrm{C} 12$ chirality is preferred in the chemical step) and our model yields energy barriers similar to those obtained with efficiently converted small molecules (further validating our proposed act $\mathrm{ACP}-a c t \mathrm{KR}$ binding mode). Further analysis of our MD simulations of $\mathbf{2}$ inside actKR identified residues (such as Gly95 and Arg220) that are important for steering the binding of the substrate and holding it 'in standby' in the actKR active site, as well as those that may aid regioselective cyclization between $\mathrm{C} 7$ and C12.

In summary, we have combined protein-protein docking, extensive MD simulation, NMR and $\mathrm{QM} / \mathrm{MM}$ reaction 
simulations to produce and validate a detailed model of the act $\mathrm{KR}-$ act $\mathrm{ACP}$ interaction that is consistent with all currently available experimental data for cyclization and ketoreduction of the natural octaketide substrate..$^{5-6}$ The model obtained provides important mechanistic insights, demonstrating the use of multiscale atomistic simulations to improve our understanding of biocatalytic protein-protein complexes. We have shown that the specificity of the actKR-act ACP interaction, together with the architecture of the $\operatorname{act} \mathrm{KR}$ active site, has direct implications for the elegant regio- and stereoselectivity of actKR towards its natural substrate. The information obtained can aid in future engineering of type II PKS ketoreductase/acyl carrier systems, e.g., to make them process alternative substrates; an important step towards building biocatalytic systems that can yield new polyketide derivatives.

\section{ASSOCIATED CONTENT}

Supporting Information. The Supporting Information is available free of charge on the ACS Publications website at DOI:

Additional details on docking calculations, construction of starting structures, parametrization of isomer-conformers of 2 , MD simulation and analysis, NMR data, details of QM/MM simulations, analysis of hydrogen bonds in MD runs in II. (PDF)

Input files for protein-protein docking; structures of M1-M20,

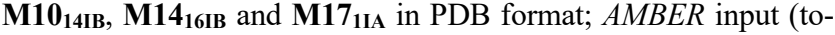
pologies and coordinates) for all runs in $\mathbf{I}_{\mathbf{A}}, \mathbf{I}_{\mathbf{B}}$, and $\mathbf{I I}$; modified force field parameters for the PPant moiety and octaketide portions of 2. (ZIP)

\section{AUTHOR INFORMATION}

\section{Corresponding Author}

* E-mail: Marc.VanderKamp@,bristol.ac.uk

\section{Present Addresses}

$\wedge$ Stefano A. Serapian: Department of Organic Chemistry, University of Pavia, viale Taramelli 10, 27100, Pavia, Italy.

\section{Orcid}

Stefano A. Serapian: 0000-0003-0122-8499

John Crosby: 0000-0002-8389-7102

Matthew P. Crump: 0000-0002-7868-5818

Marc W. van der Kamp: 0000-0002-8060-3359

\section{Author Contributions}

SAS and MWK designed the in silico experiments, SAS performed experiments, MWK devised the project. JC and MPC designed and performed the NMR experiments. SAS, MPC and MWK analyzed results and wrote the manuscript.

\section{Funding Sources}

MWK and SAS thank BBSRC for funding (David Phillips Fellowship to MWK, grant BB/M026280/1). MPC and JC also thank BBSRC for funding (grant BB/F014570/1).

\section{ACKNOWLEDGMENT}

This work was conducted using the computational facilities of the Advanced Computing Research Centre of the University of Bristol. The authors thank Prof. Shiou-Chan Tsai (Sheryl) and Gabriel O. Moreno (UC Irvine) for sharing their crystallographic data. SAS thanks Amaurys Ávila-Ibarra (University of Bristol) for his guidance with the protein-protein docking. JC and MPC thank Louise Pearson for her assistance with the NMR experiments.

\section{REFERENCES}

1. Chen, A.; Re, R. N.; Burkart, M. D., Type II Fatty Acid and Polyketide Synthases: Deciphering Protein-Protein and Protein-Substrate Interactions. Nat. Prod. Rep. 2018, 35, 1029-1045.

2. Hertweck, C.; Luzhetskyy, A.; Rebets, Y.; Bechthold, A., Type II Polyketide Synthases: Gaining a Deeper Insight into Enzymatic Teamwork. Nat. Prod. Rep. 2007, 24, 162-190.

$3 . \quad$ Zhang, Z.; Pan, H. X.; Tang, G. L., New Insights into Bacterial Type II Polyketide Biosynthesis. F1000Research 2017, 6, 172.

4. Du, D.; Katsuyama, Y.; Shin - ya, K.; Ohnishi, Y., Reconstitution of a Type II Polyketide Synthase That Catalyzes Polyene Formation. Angew. Chem. Int. Ed. 2018, 57, 1954-1957.

5. Javidpour, P.; Bruegger, J.; Srithahan, S.; Korman, Tyler P.; Crump, Matthew P.; Crosby, J.; Burkart, Michael D.; Tsai, S.-C., The Determinants of Activity and Specificity in Actinorhodin Type II Polyketide Ketoreductase. Chem. Biol. 2013, 20, 1225-1234.

6. Javidpour, P.; Korman, T. P.; Shakya, G.; Tsai, S.-C., Structural and Biochemical Analyses of Regio- and Stereospecificities Observed in a Type II Polyketide Ketoreductase. Biochemistry 2011, 50, 4638-4649.

7. Korman, T. P.; Hill, J. A.; Vu, T. N.; Tsai, S.-C., Structural Analysis of Actinorhodin Polyketide Ketoreductase: Cofactor Binding and Substrate Specificity. Biochemistry 2004, 43, 14529-14538.

8. Korman, T. P.; Tan, Y.-H.; Wong, J.; Luo, R.; Tsai, S.-C., Inhibition Kinetics and Emodin Cocrystal Structure of a Type II Polyketide Ketoreductase. Biochemistry 2008, 47, 1837-1847.

9. Javidpour, P.; Das, A.; Khosla, C.; Tsai, S.-C., Structural and Biochemical Studies of the Hedamycin Type II Polyketide Ketoreductase (HedKR): Molecular Basis of Stereo- and Regiospecificities. Biochemistry 2011, 50, 7426-7439.

10. Noey, E. L.; Tibrewal, N.; Jiménez-Osés, G.; Osuna, S.; Park, J.; Bond, C. M.; Cascio, D.; Liang, J.; Zhang, X.; Huisman, G. W.; Tang, Y.; Houk, K. N., Origins of Stereoselectivity in Evolved Ketoreductases. Proc. Natl. Acad. Sci. U. S. A. 2015, 112, E7065-E7072.

11. Tang, Y.; Lee, H. Y.; Tang, Y.; Kim, C.-Y.; Mathews, I.; Khosla, C., Structural and Functional Studies on SCO1815: A B-KetoacylAcyl Carrier Protein Reductase from Streptomyces Coelicolor A3(2). Biochemistry 2006, 45, 14085-14093.

12. Valentic, T. R.; Jackson, D. R.; Brady, S. F.; Tsai, S.-C., Comprehensive Analysis of a Novel Ketoreductase for Pentangular Polyphenol Biosynthesis. ACS Chem. Biol. 2016, 11, 3421-3430.

13. Huisman, G. W.; Liang, J.; Krebber, A., Practical Chiral Alcohol Manufacture Using Ketoreductases. Curr. Opin. Chem. Biol. 2010, 14, 122 129.

14. Moore, J. C.; Pollard, D. J.; Kosjek, B.; Devine, P. N., Advances in the Enzymatic Reduction of Ketones. Acc. Chem. Res. 2007, 40, 14121419 .

15. McDaniel, R.; Ebert-Khosla, S.; Hopwood, D. A.; Khosla, C., Rational Design of Aromatic Polyketide Natural Products by Recombinant Assembly of Enzymatic Subunits. Nature 1995, 375, 549.

16. Nivina, A.; Yuet, K. P.; Hsu, J.; Khosla, C., Evolution and Diversity of Assembly-Line Polyketide Synthases. Chem. Rev. 2019, 119, 12524-12547.

17. Neves, R. P. P.; Ferreira, P.; Medina, F. E.; Paiva, P.; Sousa, J. P. M.; Viegas, M. F.; Fernandes, P. A.; Ramos, M. J., Engineering of Pks Megaenzymes - a Promising Way to Biosynthesize High-Value Active Molecules. Top. Catal. 2021.

18. Moretto, L.; Vance, S.; Heames, B.; Broadhurst, R. W., Dissecting How Modular Polyketide Synthase Ketoreductases Interact with Acyl Carrier Protein-Attached Substrates. Chem. Commun. 2017, 53, 11457-11460.

19. Yadav, U.; Arya, R.; Kundu, S.; Sundd, M., The "Recognition Helix" of the Type II Acyl Carrier Protein (ACP) Utilizes a "Ubiquitin Interacting Motif (Uim)"-Like Surface to Bind Its Partners. Biochemistry 2018, 57, 3690-3701.

20. Colizzi, F.; Masetti, M.; Recanatini, M.; Cavalli, A., AtomicLevel Characterization of the Chain-Flipping Mechanism in Fatty-Acids Biosynthesis. J. Phys. Chem. Lett. 2016, 7, 2899-904.

21. Schiebel, J.; Chang, A.; Merget, B.; Bommineni, G. R.; Yu, W.; Spagnuolo, L. A.; Baxter, M. V.; Tareilus, M.; Tonge, P. J.; Kisker, C.; Sotriffer, C. A., An Ordered Water Channel in Staphylococcus Aureus FabI: Unraveling the Mechanism of Substrate Recognition and Reduction. Biochemistry 2015, 54, 1943-55.

22. Sulpizio, A.; Crawford, C. E. W.; Koweek, R. S.; Charkoudian, L. K., Probing the Structure and Function of Acyl Carrier Proteins to 
Unlock the Strategic Redesign of Type II Polyketide Biosynthetic Pathways. J. Biol. Chem. 2021, 296, 100328.

23. Maršavelski, A., A Novel Antimicrobial Target-Expanded and Revisited Mode of Action of Pantothenamides. RSC Adv. 2016, 6, 4488844895 .

24. Hadfield, A. T.; Limpkin, C.; Teartasin, W.; Simpson, T. J.; Crosby, J.; Crump, M. P., The Crystal Structure of the ActIII Actinorhodin Polyketide Reductase: Proposed Mechanism for ACP and Polyketide Binding. Struct. 2004, 12, 1865-1875.

25. Evans, S. E.; Williams, C.; Arthur, C. J.; Płoskoń, E.; Wattanaamorn, P.; Cox, R. J.; Crosby, J.; Willis, C. L.; Simpson, T. J.; Crump, M. P., Probing the Interactions of Early Polyketide Intermediates with the Actinorhodin ACP from S. Coelicolor A3(2). J. Mol. Biol. 2009, 389, 511528.

26. Medina, F. E.; Neves, R. P. P.; Ramos, M. J.; Fernandes, P. A., A QM/MM Study of the Reaction Mechanism of Human B-Ketoacyl Reductase. Phys. Chem. Chem. Phys. 2017, 19, 347-355.

27. Serapian, S. A.; van der Kamp, M. W., Unpicking the Cause of Stereoselectivity in Actinorhodin Ketoreductase Variants with Atomistic Simulations. ACS Catal. 2019, 9, 2381-2394.

28. Dodge, G. J.; Patel, A.; Jaremko, K. L.; McCammon, J. A.; Smith, J. L.; Burkart, M. D., Structural and Dynamical Rationale for Fatty Acid Unsaturation in Escherichia Coli. Proc. Natl. Acad. Sci. U. S. A. 2019, $116,6775-6783$

29. Milligan, J. C.; Lee, D. J.; Jackson, D. R.; Schaub, A. J.; Beld, J.; Barajas, J. F.; Hale, J. J.; Luo, R.; Burkart, M. D.; Tsai, S.-C., Molecular Basis for Interactions between an Acyl Carrier Protein and a Ketosynthase. Nat. Chem. Biol. 2019, 15, 669-671.

30. Nguyen, C.; Haushalter, R. W.; Lee, D. J.; Markwick, P. R. L.; Bruegger, J.; Caldara-Festin, G.; Finzel, K.; Jackson, D. R.; Ishikawa, F.; O’Dowd, B.; McCammon, J. A.; Opella, S. J.; Tsai, S.-C.; Burkart, M. D., Trapping the Dynamic Acyl Carrier Protein in Fatty Acid Biosynthesis. Nature 2014, 505, 427-431.

31. Rafi, S.; Novichenok, P.; Kolappan, S.; Stratton, C. F.; Rawat, R.; Kisker, C.; Simmerling, C.; Tonge, P. J., Structure of Acyl Carrier Protein Bound to FabI, the FASII Enoyl Reductase from Escherichia Coli. J. Biol. Chem. 2006, 281, 39285-39293.

32. Shen, S.; Hang, X.; Zhuang, J.; Zhang, L.; Bi, H.; Zhang, L., A Back-Door Phenylalanine Coordinates the Stepwise Hexameric Loading of Acyl Carrier Protein by the Fatty Acid Biosynthesis Enzyme BHydroxyacyl-Acyl Carrier Protein Dehydratase (Fabz). Int. J. Biol. Macromol. 2019, 128, 5-11

33. Zhang, L.; Xiao, J.; Xu, J.; Fu, T.; Cao, Z.; Zhu, L.; Chen, H.Z.; Shen, X.; Jiang, H.; Zhang, L., Crystal Structure of Fabz-ACP Complex Reveals a Dynamic Seesaw-Like Catalytic Mechanism of Dehydratase in Fatty Acid Biosynthesis. Cell Research 2016, 26, 1330-1344.

34. Masoudi, A.; Raetz, C. R. H.; Zhou, P.; Pemble Iv, C. W. Chasing Acyl Carrier Protein through a Catalytic Cycle of Lipid a Production. Nature 2014, 505, 422-426.

35. Herbst, D. A.; Huitt-Roehl, C. R.; Jakob, R. P.; Kravetz, J. M.; Storm, P. A.; Alley, J. R.; Townsend, C. A.; Maier, T., The Structural Organization of Substrate Loading in Iterative Polyketide Synthases. Nat. Chem. Biol. 2018, 14, 474-479.

36. Bräuer, A.; Zhou, Q.; Grammbitter, G. L. C.; Schmalhofer, M.; Rühl, M.; Kaila, V. R. I.; Bode, H. B.; Groll, M., Structural Snapshots of the Minimal Pks System Responsible for Octaketide Biosynthesis. Nat. Chem. 2020, 12, 755-763.

37. Fox, N. G.; Yu, X.; Feng, X.; Bailey, H. J.; Martelli, A.; Nabhan, J. F.; Strain-Damerell, C.; Bulawa, C.; Yue, W. W.; Han, S., Structure of the Human Frataxin-Bound Iron-Sulfur Cluster Assembly Complex Provides Insight into Its Activation Mechanism. Nat. Commun. 2019, 10, 2210 .

38. Kreamer, N. N. K.; Chopra, R.; Caughlan, R. E.; Fabbro, D.; Fang, E.; Gee, P.; Hunt, I.; Li, M.; Leon, B. C.; Muller, L.; Vash, B.; Woods, A. L.; Stams, T.; Dean, C. R.; Uehara, T., Acylated-Acyl Carrier Protein Stabilizes the Pseudomonas Aeruginosa Waap Lipopolysaccharide Heptose Kinase. Sci. Rep. 2018, 8, 14124.

39. Agarwal, V.; Lin, S.; Lukk, T.; Nair, S. K.; Cronan, J. E., Structure of the Enzyme-Acyl Carrier Protein (ACP) Substrate Gatekeeper Complex Required for Biotin Synthesis. Proc. Natl. Acad. Sci. U. S. A. 2012, 109, 17406-17411.

40. $\quad$ Cryle, M. J.; Schlichting, I., Structural Insights from a P450 Carrier Protein Complex Reveal How Specificity Is Achieved in the P450 Biol ACP Complex. Proc. Natl. Acad. Sci. U. S. A. 2008, 105, 1569615701.
41. McIntosh-Smith, S.; Price, J.; Sessions, R. B.; Ibarra, A. A. High Performance in Silico Virtual Drug Screening on Many-Core Processors. Int. J. High Perform. Comput. Appl. 2015, 29, 119-134.

42. $\quad$ Ellis, B. D.; Milligan, J. C.; White, A. R.; Duong, V.; Altman, P. X.; Mohammed, L. Y.; Crump, M. P.; Crosby, J.; Luo, R.; Vanderwal, C. D.; Tsai, S.-C., An Oxetane-Based Polyketide Surrogate to Probe Substrate Binding in a Polyketide Synthase. J. Am. Chem. Soc. 2018, 140, 4961-4964.

43. Li, Y.; Fiers, W. D.; Bernard, S. M.; Smith, J. L.; Aldrich, C. C; Fecik, R. A., Polyketide Intermediate Mimics as Probes for Revealing Cryptic Stereochemistry of Ketoreductase Domains. ACS Chem. Biol. 2014, 9, 2914-2922.

44. Zhao, S.; Ni, F.; Qiu, T.; Wolff, J. T.; Tsai, S. C.; Luo, R., Molecular Basis for Polyketide Ketoreductase-Substrate Interactions. Int. J. Mol. Sci. 2020, 21.

45. Søndergaard, C. R.; Olsson, M. H. M.; Rostkowski, M.; Jensen, J. H., Improved Treatment of Ligands and Coupling Effects in Empirical Calculation and Rationalization of pKa Values. J. Chem. Theory Comput. 2011, 7, 2284-2295.

46. Maier, J. A.; Martinez, C.; Kasavajhala, K.; Wickstrom, L.; Hauser, K. E.; Simmerling, C., ff14SB: Improving the Accuracy of Protein Side Chain and Backbone Parameters from ff99SB. J. Chem. Theory Comput. 2015, 11, 3696-3713.

47. Jorgensen, W. L.; Chandrasekhar, J.; Madura, J. D.; Impey, R. W.; Klein, M. L., Comparison of Simple Potential Functions for Simulating Liquid Water. J. Chem. Phys. 1983, 79, 926-935.

48. Holmberg, N.; Ryde, U.; Bulow, L., Redesign of the Coenzyme Specificity in L-Lactate Dehydrogenase from Bacillus Stearothermophilus Using Site-Directed Mutagenesis and Media Engineering. Protein Eng. 1999, 12, 851-856.

49. Wang, J.; Wolf, R. M.; Caldwell, J. W.; Kollman, P. A.; Case, D. A., Development and Testing of a General Amber Force Field. $J$. Comput. Chem. 2004, 25, 1157-1174.

50. A collection of relevant calculations is available on the ioChemBD server. https://doi.org/10.19061/iochem-bd-6-101

51. Álvarez-Moreno, M.; de Graaf, C.; López, N.; Maseras, F.; Poblet, J. M.; Bo, C., Managing the Computational Chemistry Big Data Problem: The ioChem-BD Platform. J. Chem. Inf. Model. 2015, 55, 95-103. 52. Radom, F.; Plückthun, A.; Paci, E., Assessment of Ab Initio Models of Protein Complexes by Molecular Dynamics. PLoS Comput. Biol. 2018, 14, e1006182.

53. $\quad$ Case, D. A.; Cheatham, T. E.; Darden, T.; Gohlke, H.; Luo, R.; Merz, K. M.; Onufriev, A.; Simmerling, C.; Wang, B.; Woods, R. J., The Amber Biomolecular Simulation Programs. J. Comput. Chem. 2005, 26, $1668-1688$.

54. Case, D. A.; Cerutti, D. S.; Cheatham, T. E.; Darden, T. A.; Duke, R. E.; Giese, T. J.; Gohlke, H.; Götz, A. W.; Greene, D.; Homeyer, N.; Izadi, S.; Kovalenko, A.; Lee, T. S.; Le Grand, S.; Li, P.; Lin, C.; Liu, J.; Luchko, T.; Luo, R.; Mermelstein, D.; Merz, K. M.; Monard, G.; Nguyen, H.; Omelyan, I.; Onufriev, A.; Pan, F.; Qi, R.; Roe, D. R.; Roitberg, A. E.; Sagui, C.; Simmerling, C. L.; Botello-Smith, W. M.; Swails, J. M.; Walker, R. C.; Wang, J.; Wolf, R. M.; Wu, X.; Xiao, L.; York, D. M.; Kollman, P. A. Amber 2017, University of California, San Francisco: 2017.

55. Roe, D. R.; Cheatham, T. E., PTRAJ and CPPTRAJ: Software for Processing and Analysis of Molecular Dynamics Trajectory Data. $J$. Chem. Theory Comput. 2013, 9, 3084-3095.

56. Seabra, G. d. M.; Walker, R. C.; Elstner, M.; Case, D. A.; Roitberg, A. E., Implementation of the SCC-DFTB Method for Hybrid QM/MM Simulations within the Amber Molecular Dynamics Package. $J$. Phys. Chem. A 2007, 111, 5655-5664

57. Walker, R. C.; Crowley, M. F.; Case, D. A., The Implementation of a Fast and Accurate QM/MM Potential Method in Amber. J. Comput. Chem. 2008, 29, 1019-1031.

58. Shuichi, M.; Kollman, P. A., Settle: An Analytical Version of the Shake and Rattle Algorithm for Rigid Water Models. J. Comput. Chem. 1992, 13, 952-962.

59. Stewart, J. J. P., Optimization of Parameters for Semiempirical Methods V: Modification of NDDO Approximations and Application to 70 Elements. J. Mol. Model. 2007, 13, 1173-1213.

60. Kumar, S.; Rosenberg, J. M.; Bouzida, D.; Swendsen, R. H.; Kollman, P. A., The Weighted Histogram Analysis Method for Free-Energy Calculations on Biomolecules. I. The Method. J. Comput. Chem. 1992, 13, 1011-1021. 
61. Kumar, S.; Rosenberg, J. M.; Bouzida, D.; Swendsen, R. H; Kollman, P. A., Multidimensional Free-Energy Calculations Using the Weighted Histogram Analysis Method. J. Comput. Chem. 1995, 16, 13391350 .

62. Rout, M. P.; Sali, A., Principles for Integrative Structural Biology Studies. Cell 2019, 177, 1384-1403.

63. Anand, S.; Mohanty, D., Modeling Holo-ACP:DH and HoloACP:KR Complexes of Modular Polyketide Synthases: A Docking and Molecular Dynamics Study. BMC Struct. Biol. 2012, 12, 10-20.

64. Viegas, M. F.; Neves, R. P. P.; Ramos, M. J.; Fernandes, P. A., Modeling of Human Fatty Acid Synthase and in Silico Docking of Acyl Carrier Protein Domain and Its Partner Catalytic Domains. J. Phys. Chem. $B$ 2018, 122, 77-85.

65. By "reactive pose", we mean an enzyme-substrate complex positioned such that the substrate is in place for the chemical reaction to start; others have recently referred to this as a "near attack conformation" or NAC.
66. Di Maio, G.; Mascia, M. G.; Vecchi, E., Does Substituent's Conformation Influence the Kinetics of Reduction Reactions on Trans-4 X-Decal-1-Ones and to What Extent? Tetrahedron 2002, 58, 3313-3318.

67. Wu, Y. D.; Tucker, J. A.; Houk, K. N., Stereoselectivities of Nucleophilic Additions to Cyclohexanones Substituted by Polar Groups. Experimental Investigation of Reductions of Trans-Decalones and Theoretical Studies of Cyclohexanone Reductions. The Influence of Remote Electrostatic Effects. J. Am. Chem. Soc. 1991, 113, 5018-5027.

68. Østergaard, L. H.; Kellenberger, L.; Cortés, J.; Roddis, M. P. Deacon, M.; Staunton, J.; Leadlay, P. F., Stereochemistry of Catalysis by the Ketoreductase Activity in the First Extension Module of the Erythromycin Polyketide Synthase. Biochemistry 2002, 41, 2719-2726. 